Chin-Chung Shu

Chih-Hsin Lee

Jann-Yuan Wang

Jih-Shuin Jerng

Chong-Jen Yu

Po-Ren Hsueh

Li-Na Lee

Pan-Chyr Yang

the TAMI group

\section{Nontuberculous mycobacteria pulmonary infection in medical intensive care unit: the incidence, patient characteristics, and clinical significance}

Received: 31 March 2008

Accepted: 7 July 2008

Published online: 22 July 2008

(C) The Author(s) 2008

Taiwan Anti-Mycobacteria Investigation (TAMI) group: J.-Y. Wang, L.-N. Lee, C.-J. Yu, P.-C. Yang, W.-J. Su, C.-C. Shu, H.-C. Lai, C.-H. Lee and M.-C. Yu.

\section{C.-C. Shu}

Department of Internal Medicine, National Taiwan University Hospital, Yun-Lin Branch, Yun Lin, Taiwan

\section{C.-H. Lee}

Department of Internal Medicine, Buddhist Tzu Chi General Hospital, Taipei Branch, Taipei, Taiwan

\section{J.-Y. Wang (凶)}

Department of Internal Medicine, National Taiwan University Hospital, \# 7, Chung-Shan South Road,

Taipei 100, Taiwan

e-mail: jywang@ntu.edu.tw

Tel.: +886-2-23562905

Fax: +886-2-23582867

J.-S. Jerng - C.-J. Yu · P.-C. Yang Department of Internal Medicine, National Taiwan University Hospital, Taipei, Taiwan

\section{P.-R. Hsueh · L.-N. Lee} Department of Laboratory Medicine, National Taiwan University Hospital, Taipei, Taiwan

Abstract Background: The clinical significance of nontuberculous mycobacteria (NTM) pulmonary infection in medical intensive care unit (ICU) is still unclear. Materials and methods: We conducted a retrospective study in the medical ICUs of a medical center in Taiwan from January 1999 to June 2007. Patients with NTM isolated from respiratory specimens within 1 month before or during the ICU course were identified. Those who fulfilled the diagnostic criteria of NTM pulmonary infection were identified and compared with patients with NTM colonization and control subjects who were culture-negative for mycobacteria. Results: Among the 5,378 patients admitted to medical ICUs, $2,866(53.3 \%)$ had received mycobacterial culture for respiratory specimens. NTM were isolated from $169(5.8 \%)$ patients. Of them, 47 $(27.8 \%)$ were considered NTM pulmonary infection. $M$. avium complex and $M$. abscessus were the most common pathogens. Within 100 days after ICU admission, significantly more patients with NTM infection died than those with NTM colonization and control subjects (47 vs. 8 vs. $14 \%, P<0.001)$. Twenty-one $(49 \%)$ patients with NTM pulmonary infection received anti-NTM treatment, with four experiencing adverse effects. Although statistically insignificant, anti-NTM treatment was associated with prolonged survival for those who died in the ICU and shorter ICU stay for those who survived the ICU course.

Conclusion: Our findings suggest that NTM pulmonary infection seems to associate with higher mortality in medical ICUs. Anti-NTM treatment is probably associated with a better outcome. Therefore, keeping a high suspicion when NTM is isolated and using careful consideration when starting anti-NTM treatment should be emphasized.

Keywords Nontuberculous mycobacteria $\cdot$ Pulmonary infection . Intensive care unit

\section{Introduction}

Unlike Mycobacterium tuberculosis (Mtb), nontuberculous mycobacteria (NTM) exists in the environment and can be isolated from clinical specimens due to true infection, colonization, or even contamination [1]. It is an important clinical concern since the rate of NTM pulmonary infection has increased over the last 10 years 
[2-4]. The reasons for this increase are not clear, but probably result from an enlarged population of acquired immunocompromised individuals, chronic lung disease, and prolonged mechanical ventilation [2, 5-9]. In our hospital, among the patients with acid-fast smear-positive respiratory specimens in 2006, 35\% were due to NTM [10]. In fact, NTM pulmonary infection remains a diagnostic challenge even under the contemporary guidelines of the American Thoracic Society [1]. Because the presence of NTM in respiratory specimens is not pathognomonic for NTM infection, treatment is not deemed necessary by every clinician [11]. Even in disseminated NTM disease, a previous study revealed that adequate treatment was not started until 130 days after the initial visit [12]. However, the mortality rate can exceed $50 \%$ within 5 years $[11,13,14]$.

In patients with complicated and critical conditions, such as those admitted to intensive care unit (ICU), the clinical significance of NTM in respiratory specimens and the prognostic impact of NTM pulmonary infection are even more difficult to understand than in stable patients $[11,15]$. Therefore, we conducted a retrospective study including all medical ICU patients with NTM being isolated from respiratory specimens within a period of 8.5 years to evaluate the clinical significance of the presence of NTM and compare the demographic characteristics, clinical manifestations, and outcome in patients with NTM pulmonary infection with those with NTM colonization and control subjects whose respiratory samples were culture-negative for mycobacteria.

\section{Materials and methods}

Subject of study

This study was conducted in National Taiwan University Hospital, a tertiary-care referral center with 2,150 beds in northern Taiwan, and was approved by the Institutional Review Boards of the hospital. We reviewed the mycobacterial laboratory registry database and identified all patients who had undergone mycobacterial culture for respiratory specimens between January 1999 and June 2007. Among them, only those with the mycobacterial culture performed within 1 month before or during admission to medical ICUs were included for further analysis. In our hospital, mycobacterial culture for respiratory specimens is usually ordered in the following three conditions: (1) typical radiographic findings of postprimary pulmonary tuberculosis, i.e. patchy, poorly defined consolidation particularly in the apical and posterior segments of the upper lobes and with cavitation [16]; (2) contact history of active tuberculosis [17]; or (3) a poor clinical response to anti-bacterial antibiotics, defined as no improvement of symptoms, radiographic findings, or laboratory data within 3 days after antibiotics
[18]. Mycobacterial culture was performed as previously described [19]. Mycobacterial species were identified by using biochemical testing [20].

\section{Selection criteria of patients}

In this study, NTM pulmonary infection was considered definite if all the following were met [1]: (1) at least two respiratory specimens or one bronchial washing/brushing sample being culture-positive for the same NTM species; (2) presence of respiratory symptoms; (3) chest radiography or computed tomography demonstrating new patch(es) of consolidation, nodular infiltrates, cavitary lesions, or multifocal bronchiectasis; and (4) no bacterial pathogens being isolated from respiratory specimens at the same time. NTM pulmonary infection was considered probable if a patient fulfilled the second and third criteria of definite NTM and had three respiratory specimens that yielded the same NTM species, yet bacterial pathogens were isolated at the same time. The definite and probable groups were considered as NTM pulmonary infection. Because the presence of NTM in respiratory samples can result from environmental contamination, only patients who fulfilled the first criterion of microbiology but were not considered to have NTM pulmonary infection were considered as NTM colonization (colonization group) [1]. Those who had a radiographic response to anti-bacterial antibiotics were classified into NTM colonization even if they had two respiratory samples that were culture-positive for the same NTM. For each patient with NTM pulmonary infection, an age-, gender-, and APACHE II score-matched control subject for whom mycobacterial culture was performed during ICU admission for $\geq 3$ respiratory samples with the results being all negative was selected as control group.

\section{Clinical data}

The medical records were reviewed. The chest images were noted as in our previous study [21]. Radiographically, presence of reticulonodular lesions compatible with pulmonary fibrosis and multifocal bronchiectasis were categorized as structural lung change. The adequacy of anti-NTM treatment was judged according to the ATS guidelines [1]. Patients were followed for 100 days after ICU admission or until death or discharge from the hospital.

\section{Statistics}

The inter-group differences were compared by using OneWay ANOVA for numerical variables and chi-square test for categorical variables. Adjustments for multiple 
comparisons were made by applying the Bonferonni method. Survival curves within 100 days since ICU admission for each class of variables were generated using the Kaplan-Meier method and were compared using the log-rank test. Variables having a significant difference $(P<0.05)$ were further tested by multivariate Cox proportional hazard regression analysis.

\section{Results}

From January 1999 to June 2007, a total of 4,779 respiratory specimens were culture-positive for NTM (Table 1). During this period, a total of 5,378 patients were admitted to our medical ICUs. Of them, 2,866 $(53.3 \%)$ received mycobacterial culture of respiratory specimens. NTM was isolated from 337 samples from 169 patients collected within 1 month before or during admission to the medical ICUs. Of them, $47(27.8 \%)$ had NTM pulmonary infection $(24(14.2 \%)$ definite and 23 $(13.6 \%)$ probable), and 25 (14.8\%) had NTM colonization. Mycobacterium avium complex was the most common NTM species. The medical records were not available for four patients with NTM pulmonary infection and one in the colonization group. Therefore, a total of 43 patients with NTM pulmonary infection, 24 with NTM pulmonary colonization, and another 43 age-, sex-, and APACHE-II score-matched control subjects were further investigated.

\section{Clinical data}

Statistically, no significant difference was noted in the age, gender, and cause of ICU admission among the three groups (Table 2). Previous tracheostomy was significantly associated with the colonization group $(P<0.001)$. Structural lung change was predominantly found in patients with NTM pulmonary infection $(P<0.001)$. Underlying co-morbid condition was more frequent in the control group $(P=0.015)$. The most common was malignancy in the NTM infection group, but diabetes mellitus in the other two groups. HIV serostatus was checked in $15(35 \%), 10(42 \%)$, and $10(42 \%)$ patients in the NTM infection, NTM colonization, and control groups, respectively. The prevalence of HIV/ AIDS was not significantly different. Respiratory failure was the most common indication for ICU admission in all groups.

Chronic obstructive pulmonary disease (COPD) was previously diagnosed in $10(23.3 \%)$ patients with NTM infection, $5(20.8 \%)$ with NTM colonization, and 7 $(16.3 \%)$ in the control group. The prevalence was not significantly different among the three groups $(P=0.722)$. Among patients with NTM infection, pulmonary function test was performed in eight and revealed severe obstructive in 2 , mild obstructive in 1 , severe restrictive in 2 , moderate restrictive in 1 , mixed obstructive and restrictive defect in 1, and normal in the remaining one. Two patients with NTM colonization underwent pulmonary function test. The results were moderate obstructive in 1 and severe restrictive in another. Univariate analysis revealed that COPD did not associate with 100-days mortality in ICU $(P=0.989)$.

Radiographically, there were significant differences among the NTM infection, NTM colonization, and control groups in the prevalence of pulmonary consolidation (31 $(72 \%)$ vs. $11(46 \%)$ vs. $32(74 \%), P=0.04)$, and reticulonodular shadowing $(23(51 \%)$ vs. $2(8 \%)$ vs. 4 (9\%), $P<0.001)$. Of the colonization group, $5(21 \%)$ patients had negative radiographic findings. In the NTM infection group, $15(35 \%)$ patients with reticulonodular shadowing had cavitary lesions, whereas none in the other two groups did.

At the beginning of the ICU course, the APACHE II scores (Table 2), hemogram, and biochemistry, was not significantly different among the NTM infection, NTM colonization, and control groups. However, the control group had the highest serum creatinine $(1.2 \pm 0.7$ vs. $1.5 \pm 1.4$ vs. $2.3 \pm 2.1 \mathrm{mg} / \mathrm{dl}, P=0.009)$ and ratio of arterial oxygen pressure divided by fraction of supplemental

Table 1 Number of isolates and patients with different NTM species in different clinical conditions during the 8.5 years

\begin{tabular}{|c|c|c|c|c|c|c|}
\hline \multirow{3}{*}{$\begin{array}{l}\text { Mycobacterial } \\
\text { species }\end{array}$} & \multicolumn{5}{|c|}{ Numbers of NTM isolates and patients in intensive care unit } & \multirow{3}{*}{$\begin{array}{l}\text { All isolates in } \\
\text { our hospital } \\
(N=4,779)\end{array}$} \\
\hline & \multicolumn{2}{|l|}{ NTM infection } & \multicolumn{2}{|l|}{ NTM colonization } & \multirow{2}{*}{$\begin{array}{l}\text { Contamination } \\
\text { Patients }(N=97)\end{array}$} & \\
\hline & Isolates $(N=175)$ & Patients $(N=47)$ & Isolates $(N=65)$ & Patients $(N=25)$ & & \\
\hline M. avium complex & $67(38.3 \%)$ & $20(42.6 \%)$ & $27(41.5 \%)$ & $11(44 \%)$ & $33(34 \%)$ & $1,527(31.9 \%)$ \\
\hline M. abscessus & $29(16.6 \%)$ & $8(17 \%)$ & $24(36.9 \%)$ & $8(32 \%)$ & $13(13.4 \%)$ & $898(18.8 \%)$ \\
\hline M. chelonae & $34(19.4 \%)$ & $7(14.9 \%)$ & $4(6.2 \%)$ & $2(8 \%)$ & $2(2.19 \%)$ & $546(11.4 \%)$ \\
\hline M. fortuitum & $10(5.7 \%)$ & $3(6.4 \%)$ & $7(10.8 \%)$ & $2(8 \%)$ & $13(13.4 \%)$ & $646(13.5 \%)$ \\
\hline M. kansasii & $27(15.4 \%)$ & $7(14.9 \%)$ & $2(3.1 \%)$ & $1(4 \%)$ & $13(13.4 \%)$ & $310(6.5 \%)$ \\
\hline Others & $8(4.6 \%)$ & $2(4.3 \%)$ & $1(1.5 \%)$ & $1(4 \%)$ & $23(23.7 \%)$ & $852(17.8 \%)$ \\
\hline
\end{tabular}


Table 2 Clinical characteristics

\begin{tabular}{llll}
\hline & NTM infection $(N=43)$ & NTM colonization $(N=24)$ & Matched control $(N=43)$ \\
\hline Age: year, mean (range) & $69.7(19-95)$ & $67.7(28-91)$ & $69.8(19-96)$ \\
Age > 65 & $28(65 \%)$ & $16(67 \%)$ & $28(65 \%)$ \\
Male gender & $25(58 \%)$ & $20(83 \%)$ & $25(58 \%)$ \\
Structural lung change*,a & $25(58 \%)$ & $5(21 \%)$ & $5(12 \%)$ \\
Having received tracheostomy* & $7(16 \%)$ & $10(42 \%)$ & $4(9 \%)$ \\
Underlying co-morbid condition* & $19(44 \%)$ & $8(33 \%)$ & $29(67 \%)$ \\
Malignancy & $14(33 \%)$ & $2(8 \%)$ & $8(19 \%)$ \\
AIDS & $2(5 \%)$ & $2(8 \%)$ & $2(5 \%)$ \\
Diabetes mellitus & $2(5 \%)$ & $3(13 \%)$ & $11(33 \%)$ \\
End-stage renal disease & $1(2 \%)$ & $1(4 \%)$ & $4(12 \%)$ \\
Cirrhosis of liver & $2(5 \%)$ & 0 & 0 \\
Autoimmune disease & 0 & 0 & $4(9 \%)$ \\
Cause of ICU admission & $35(81 \%)$ & $18(75 \%)$ & $29(67 \%)$ \\
Respiratory failure & $32(74 \%)$ & $15(63 \%)$ & $28(65 \%)$ \\
Pneumonia & $1(2 \%)$ & 0 & $1(2 \%)$ \\
COPD with AE & $2(5 \%)$ & $2(8 \%)$ & 0 \\
Pneumothorax & $5(12 \%)$ & $1(4 \%)$ & $10(23 \%)$ \\
Sepsis, other than pneumonia & $1(2 \%)$ & 0 & 0 \\
Seizure & $1(2 \%)$ & $1(4 \%)$ & 0 \\
Attempted suicide & $0(2 \%)$ & $2(8 \%)$ & $2(5 \%)$ \\
DKA/NKHS & $1(2 \%)$ & $19.1 \pm 8.0$ & $2(5 \%)$ \\
Cardiovascular disease & $22.8 \pm 6.9$ & $20.2 \pm 6.6$ & $2.8 \pm 7.8$ \\
APACHE II-initial (mean \pm SD) & & $21.2 \pm 7.5$ \\
APACHE II-24 h (mean \pm SD) & 20.6 &
\end{tabular}

Data are no. (\%), unless otherwise indicated

$A I D S$ acquired immunodeficiency syndrome; $A P A C H E$ acute physiology and chronic healthy evaluation; $C O P D$ with $A E$ chronic obstructive pulmonary disease with acute exacerbation; $D K A$ diabetic ketotic acidosis; NKHS non-ketotic hyperosmolar syndrome
* Significant difference $(P<0.05)$ between the three groups

a Structural lung change was considered if reticulonodular lesions compatible with pulmonary fibrosis and multifocal bronchiectasis were present on chest images oxygen concentration $\left(\mathrm{PaO}_{2} / \mathrm{FiO}_{2}\right)(252.2 \pm 107$ vs. 247.4 \pm 119 vs. $337 \pm 168 \mathrm{mmHg}, P=0.001)$, and the lowest arterial pressure of carbon dioxide $\left(\mathrm{PaCO}_{2}\right)(53.8 \pm 23.4$ vs. $57.3 \pm 26.9$ vs. $40.2 \pm 11.8 \mathrm{mmHg}, P=0.002)$.

\section{Treatment and outcome}

Table 3 and Fig. 1 show that the ICU-mortality and 100days mortality were highest in the NTM infection group.
Though statistically insignificant, the weaning rate was highest in the control group. Univariate survival analysis revealed that NTM pulmonary infection was associated with a poor outcome $(P=0.008$; hazard ratio $(\mathrm{HR})$ : 7.2 ; 95\% CI: 1.7-30.8). To exclude the potential confounding effect on NTM infection for survival, a multivariate Cox regression analysis, containing other variables that were significantly different among the three groups during univariate analysis were performed. These variables included NTM infection, structural lung change, tracheostomy, and

Table 3 Outcome of patients

\begin{tabular}{|c|c|c|c|c|}
\hline & \multicolumn{2}{|l|}{ NTM infection } & \multirow{2}{*}{$\begin{array}{l}\text { NTM colonization } \\
\text { All }(N=24)\end{array}$} & \multirow{2}{*}{$\begin{array}{l}\text { Control } \\
\text { All }(N=43)\end{array}$} \\
\hline & Untreated $(N=22)$ & Treated $(N=21)$ & & \\
\hline Weaning rate & $8(n=19,42 \%)$ & $6(n=16,38 \%)$ & $9(n=18,50 \%)$ & $27(n=37,73 \%)$ \\
\hline MV day: days \pm SD & $6.25 \pm 3.1$ & $10.2 \pm 10.2$ & $16.0 \pm 9.6$ & $11.33 \pm 12.3$ \\
\hline ICU-mortality* & $5(26 \%)$ & $6(29 \%)$ & $2(8 \%)$ & $2(5 \%)$ \\
\hline ICU-stay: days \pm SD & $21.4 \pm 15.7$ & $22.4 \pm 16.4$ & $20.7 \pm 19.3$ & $18.0 \pm 15.2$ \\
\hline Survived: days \pm SD & $22.7 \pm 16.4$ & $18 \pm 11.4$ & $17 \pm 14.8$ & $18.0 \pm 15.3$ \\
\hline Dead: days $\pm \mathrm{SD}$ & $17.0 \pm 13.7$ & $33.3 \pm 22.5$ & $61 \pm 21.2$ & $18.1 \pm 15.1$ \\
\hline 100-days mortality* & $10(45.6 \%)$ & $10(47.6 \%)$ & $2(8.3 \%)$ & $6(14 \%)$ \\
\hline
\end{tabular}

Data are no. (\%), unless otherwise indicated

$I C U$ intensive care unit; $M V$ mechanical ventilation; $N T M$ nontuberculous mycobacteria

* Significant difference $(P<0.05)$ between NTM infection, NTM colonization and control groups 


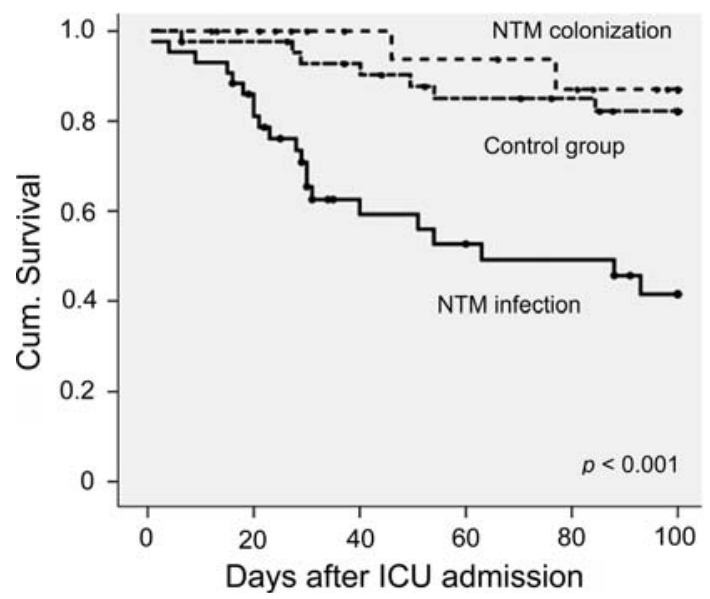

Fig. 1 Survival curve for patients with NTM pulmonary infection, colonization, or control group plotted using the Kaplan-Meier method. Black dots represent patients who were still alive at the end of the study

underlying co-morbid conditions. The results revealed NTM pulmonary infection still had significant prognostic impact ( $P=0.004$; HR: 8.7 ; 95\% CI: $2.0-38.5)$, and none of the other three variables was an independent prognostic factor (structural lung change: $\mathrm{HR}=1.6,95 \% \mathrm{CI}$ : $0.7-3.7$; tracheostomy: $\mathrm{HR}=0.9,95 \% \mathrm{CI}: 0.3-2.3$; underlying comorbid condition: $\mathrm{HR}=0.7,95 \% \mathrm{CI}: 0.3-1.5$ ).

In the NTM infection group, mycobacterial culture for respiratory samples was performed prior to ICU admission in 31 patients, and was positive for NTM in 13. The 13 patients had a higher 100-day mortality rate than the 30 patients from whom NTM was isolated after ICU admission $(61.5$ vs. $40 \%, P=0.025)$. More patients in the latter had underlying co-morbid condition than in the former ( 23 vs. $53 \%, P=0.098$ ). Other clinical characteristics, such as APACHE II score (21.55 vs. 23.4), albumin $(3.28$ vs. $3.08 \mathrm{~g} / \mathrm{dl})$, serum creatinine $(1.0 \mathrm{vs}$. $1.3 \mathrm{mg} / \mathrm{dl})$, and C-reactive protein (13.1 vs. $10.4 \mathrm{mg} / \mathrm{dl})$ were similar in the two subgroups.

Of the 22 patients in the NTM infection or NTM colonization group who died within 100 days after ICU admission, nine patients, including two belonging to the colonization group, had definite causes of death other than NTM infection. Another ten died of respiratory failure due to refractory pneumonia with unknown pathogens. The remaining three patients died of multi-organ failure, one of them having $M$. avium-intracellular complex (MAC) bacteremia. Among the ten patients with NTM infection who were treated and died within 100 days after ICU admission, all had multidrug-resistant enterobacteriae such as Pseudomonas aeruginosa or Acinetobacter baumannii, defined as resistance to $\geq 2$ classes of antipseudomonal agents, or methicillin-resistant Staphylococcus aureus, isolated from their respiratory samples during the following hospital course.
Of the patients with NTM pulmonary infection, treatment for NTM was prescribed in 21 (49\%), and was started $21.1 \pm 28.3$ days on average after ICU admission. Although statistically insignificant, anti-NTM treatment was associated with prolonging survival for those who died in the ICU and with shortening the ICU stay for those who survived the ICU course (Table 3 ). All eight patients with MAC infection were treated with macrolides-containing regimens, with four not receiving ethambutol. Four of ten patients who were treated for rapid growing mycobacteria infection received macrolide monotherapy. The remaining three received treatment for $M$ kansasii or $M$. terrae infection according to the ATS guidelines. Five episodes of adverse effects were reported in four patients, including one each for bone marrow suppression, hepatitis, diarrhea, fever and blurred vision.

\section{Discussion}

The 5-year mortality rate has been reported to be around $34-69 \%$ in NTM pulmonary infection $[11,14,22]$. However, decision making for treating NTM is a big clinical challenge because NTM are usually considered as colonizers rather than true pathogens in the respiratory tract. In addition, NTM pulmonary infection was usually considered to have chronic indolent disease [1, 23]. Consequently, treatment for NTM is usually delayed. The clinical significance and prognostic impact of NTM in respiratory specimens of patients in medical ICUs is even more obscure, because the issue has been discussed only in few case reports [24-26]. Therefore, decision making for treating NTM in critically-ill patients is extremely difficult. This is probably the reason why anti-NTM treatment was prescribed in only $49 \%$ of our NTM infected patients, lower than that reported in non-critical populations $(65.5 \%)$ [11].

Though the total number of the mortality cases was not sufficiently large for conducting a multivariate Cox proportional hazard regression analysis including four variables [27], our results revealed that NTM pulmonary infection was probably associated with higher mortality in medical ICUs, even after controlling the possible confounding factors. The reasons are not clear, but this is probably because NTM pulmonary infection acts as an underlying co-morbid condition, which in turn deteriorates the pulmonary function and compromises the defense mechanism of the lung, and is associated with difficult eradicating of gram-negative organisms in airway [28]. Another possible explanation is that the prolonged antibiotic treatment including new fluoroquinolone and aminoglycosides for NTM infection could probably induce the emergency of multidrug-resistant $P$. aeruginosa or A. baumannii later in the hospital course, which has been 
documented to increase the infection-related mortality [2931]. However, these issues should be further confirmed.

In ICU patients with NTM pulmonary infection, our analysis revealed that the presence of NTM in respiratory samples prior to ICU admission was associated with a worse survival. The finding that $60 \%$ (18 in 30) of those with NTM isolated during ICU course did not have NTM prior to ICU admission implies that most of them could be in an earlier course of the NTM infection and probably had better lung function and host defense.

Among non-critical patients with NTM isolated from their respiratory specimens, the proportion of true infection was $20-25 \%$ in the 2000s [15, 32]. In our study, NTM was isolated from respiratory specimens in $5.8 \%$ of all patients admitted to our medical ICUs. Among them, $27.8 \%$ were considered infection rather than colonization or contamination. The reasons for the enlarging proportion of true infection are still unclear, but probably due to the improvement of the culture technique for NTM and the higher suspicion. In addition, a growing population of chronic lung disease, prolonged mechanical ventilation and immune dysfunction due to the underlying condition, such as AIDS and malignancy has been proposed [2, 6-9]. This finding emphasizes that careful interpretation of the results of mycobacterial culture for respiratory specimens and repeated culture surveillance are extremely important in this population.

The most common species in NTM pulmonary infection were different among different studies; $M$. kansasii in England and Wales, M. xenopi in Southeast England, M. avium complex (MAC) in the United States, Japan, and South Korea, and MAC and M. abscessus in our patients [2, 4, 15, 33-35]. In contrast to previous reports, a higher proportion of rapid growing mycobacteria were found in our series (38 vs. $10 \%$ in the United States and less than $5 \%$ in Japan) $[4,33,35]$. In our study, the presence of M. chelonae isolate in the respiratory tract was more likely to be a true infection than other species (64 vs. 17-34\%) although statistically insignificant. Therefore, understanding the local epidemiology is important in the management of patients with NTM isolated from their respiratory specimens.

Radiographically, a much smaller proportion $(51 \%)$ of our patients with NTM infection had reticulonodular opacity than those (70-90\%) in previous reports $[15,36$, 37]. In contrast, there was a markedly higher proportion $(72 \%)$ of our patients with NTM infection having pulmonary consolidation than that $(30 \%)$ in the study performed by Koh et al. [15]. One possible explanation is that about half of our patients with NTM infection had underlying immunocompromised conditions, probably predisposing to other radiographic manifestations, as it has been reported that only $6 \%$ of AIDS patient with NTM pulmonary infection demonstrated typical reticulonodular opacity [38]. In addition, decreased serum albumin level, immobilization, positive pressure ventilation, and severe pulmonary inflammation in critical illness all predispose to the accumulation of pleural effusion, leading to misinterpretation for the pulmonary lesions as consolidation.

Some studies on non-critical patients have revealed that anti-NTM treatment did not improve the outcome $[11,14]$. It might be attributed to the small number of patients (31 and 42 in the two studies, respectively) and the presence of confounding factors, such as underlying co-morbid conditions. In addition, the susceptibility tests were usually not routinely performed [39] and the chemotherapy regimens for all NTM species were impossible to be standardized. In contrast, studies on AIDS patients with non-disseminated NTM disease have showed that anti-NTM treatment was beneficial in 2-year follow-up $[38,40]$. In our study, treatment for NTM infection also did not improve the outcome. However, among the patients with NTM infection who survived the ICU course, those receiving anti-NTM treatment was associated with a shorter stay in ICU. In addition, among the patients with NTM infection who died in the ICU, antiNTM treatment was associated with prolong survival (Table 3). Further large-scale prospective studies are necessary to investigate the impact of anti-NTM treatment and to clarify possible confounding factors.

If anti-NTM treatment does improve the outcome of patients in ICU, keeping a high suspicion, establishing the diagnosis early, and starting adequate treatment promptly become very important. Several clinical characteristics were associated with NTM pulmonary infection, including structural lung change without tracheostomy, radiographically reticulonodular shadowing with cavitation, and isolation of $M$. chelonae. For those patients, NTM pulmonary infection should be suspected once having NTM isolated from their respiratory specimens.

Our study has several limitations. First, due to the small number of patients, we could not make any firm conclusions, especially in identifying the reasons for the high mortality rate in NTM infected patients and in analyzing the importance of anti-NTM treatment. Second, in this retrospective study, the number of patients with NTM infection could be underestimated because mycobacterial cultures were performed in $53.3 \%$ of ICU patients. In addition, structural lung change that was only mild and beyond the detection threshold of the chest radiography will be missed. Large scale prospective study is needed for further investigation.

\section{Conclusion}

Our findings suggest that NTM pulmonary infection probably increases the mortality of the medical ICU 
patients. Anti-NTM treatment could possibly improve the outcome. At present, keeping a high suspicion when NTM is isolated and using careful consideration when starting anti-NTM treatment should be emphasized.
Open Access This article is distributed under the terms of the Creative Commons Attribution Noncommercial License which permits any noncommercial use, distribution, and reproduction in any medium, provided the original author(s) and source are credited.

\section{References}

1. Griffith DE, Aksamit T, Brown-Elliott BA, Catanzaro A, Daley C, Gordin F, Holland SM, Horsburgh R, Huitt G, Iademarco MF, Iseman M, Olivier K, Ruoss S, von Reyn CF, Wallace RJ Jr, Winthrop K (2007) An official ATS/ IDSA statement: diagnosis, treatment, and prevention of nontuberculous mycobacterial diseases. Am J Respir Crit Care Med 175:367-416

2. Martin-Casabona N, Bahrmand AR, Bennedsen J, Thomsen VO, Curcio M, Fauville-Dufaux M, Feldman K, Havelkova M, Katila ML, Koksalan K, Pereira MF, Rodrigues F, Pfyffer GE, Portaels F, Urgell JR, Rusch-Gerdes S, Tortoli E, Vincent V, Watt B (2004) Non-tuberculous mycobacteria: patterns of isolation. A multi-country retrospective survey. Int J Tuberc Lung Dis 8:1186-1193

3. Field SK, Cowie RL (2006) Lung disease due to the more common nontuberculous mycobacteria. Chest 129:1653-1672

4. Marras TK, Daley CL (2002) Epidemiology of human pulmonary infection with nontuberculous mycobacteria. Clin Chest Med 23:553567

5. Miguez-Burbano MJ, Flores M, Ashkin D, Rodriguez A, Granada AM, Quintero N, Pitchenik A (2006) Non-tuberculous mycobacteria disease as a cause of hospitalization in HIV-infected subjects. Int J Infect Dis 10:47-55

6. Chetchotisakd P, Kiertiburanakul S, Mootsikapun P, Assanasen S, Chaiwarith R, Anunnatsiri S (2007) Disseminated nontuberculous mycobacterial infection in patients who are not infected with HIV in Thailand. Clin Infect Dis 45:421-427

7. Saritsiri S, Udomsantisook N, Suankratay C (2006) Nontuberculous mycobacterial infections in King Chulalongkorn Memorial Hospital. J Med Assoc Thai 89:2035-2046

8. Pilcher DV, Bailey MJ, Treacher DF, Hamid S, Williams AJ, Davidson AC (2005) Outcomes, cost and long term survival of patients referred to a regional weaning centre. Thorax 60:187-192
9. Dailloux M, Abalain ML, Laurain C, Lebrun L, Loos-Ayav C, Lozniewski A, Maugein J (2006) Respiratory infections associated with nontuberculous mycobacteria in non-HIV patients. Eur Respir J 28:1211-1215

10. Jann-Yuan Wang P-RH, Chong-Jen Yu, I-Shiow Jan, Li-Na Lee, Pan-Chyr Yang (2007) Cause of Sputum AcidFast Smear-Positive: Mycobacterium tuberculosis or nontuberculous mycobacterium. Chest doi: http://www.chestjournal.org/cgi/ eletters/129/6/1653

11. Andrejak C, Lescure FX, Douadi Y, Laurans G, Smail A, Duhaut P, Jounieaux V, Schmit JL (2007) Nontuberculous mycobacteria pulmonary infection: management and follow-up of 31 infected patients. J Infect 55:3440

12. Lai CC, Lee LN, Ding LW, Yu CJ, Hsueh PR, Yang PC (2006) Emergence of disseminated infections due to nontuberculous mycobacteria in nonHIV-infected patients, including immunocompetent and immunocompromised patients in a university hospital in Taiwan. J Infect 53:77-84

13. Research Committee, British Thoracic Society (1994) Mycobacterium kansasii pulmonary infection: a prospective study of the results of nine months of treatment with rifampicin and ethambutol. Research Committee, British Thoracic Society. Thorax 49:442-445

14. Jenkins PA, Campbell IA (2003) Pulmonary disease caused by Mycobacterium xenopi in HIV-negative patients: five year follow-up of patients receiving standardised treatment. Respir Med 97:439-444

15. Koh WJ, Kwon OJ, Jeon K, Kim TS, Lee KS, Park YK, Bai GH (2006) Clinical significance of nontuberculous mycobacteria isolated from respiratory specimens in Korea. Chest 129:341348

16. Andreu J, Caceres J, Pallisa E, Martinez-Rodriguez M (2004) Radiological manifestations of pulmonary tuberculosis. Eur J Radiol 51:139-149
17. Menzies D, Joshi R, Pai M (2007) Risk of tuberculosis infection and disease associated with work in health care settings. Int J Tuberc Lung Dis 11:593605

18. Hidayat LK, Hsu DI, Quist R, Shriner KA, Wong-Beringer A (2006) Highdose vancomycin therapy for methicillin-resistant Staphylococcus aureus infections: efficacy and toxicity. Arch Intern Med 166:2138-2144

19. Wang JY, Hsueh PR, Wang SK, Jan IS, Lee LN, Liaw YS, Yang PC, Luh KT (2007) Disseminated tuberculosis: a 10year experience in a medical center. Medicine 86:39-46

20. Pfyffer GE, Vicent V, Christina Gutiérrez M, Brown-Elliott BA, Wallace RJ (2007) Myobacterium. In: Murray PR, Baron EJ, Jorgensen JH, Pfaller MA, Landry ML (eds) Manual of Clinical Microbiology, 9th ed. American Society for Microbiology, Washington, DC, pp 543-600

21. Wang JY, Hsueh PR, Jan IS, Lee LN, Liaw YS, Yang PC, Luh KT (2006) Empirical treatment with a fluoroquinolone delays the treatment for tuberculosis and is associated with a poor prognosis in endemic areas. Thorax 61:903-908

22. Society TRCotBT (2003) Pulmonary disease caused by $M$. malmoense in HIV negative patients: 5-yr follow-up of patients receiving standardised treatment. Eur Respir J 21:478-482

23. Tanaka E (1998) Clinical features and diagnosis of disease caused by nontuberculous mycobacteria. Nippon Rinsho 56:3195-3198

24. Lawn SD (2005) Acute respiratory failure due to Mycobacterium kansasii infection: immune reconstitution disease in a patient with AIDS. J Infect 51:339-340

25. Miyachi S, Kosuda T, Masai H, Hidaka $\mathrm{N}$, Takizawa H, Hisatomi T (1985) A case of intractable pulmonary infection due to Mycobacterium fortuitum resulting in respiratory failure. Kekkaku 60:277-281 
26. Yamada H, Kohyama T, Terashi K, Mori H, Yamaguchi A, Arima N, Tanaka H (1997) Fatal pulmonary infection due to multidrug-resistant Mycobacterium kansasii which developed in an immunocompetent young man. Intern Med 36:298-300

27. Peduzzi P, Concato J, Feinstein AR, Holford TR (1995) Importance of events per independent variable in proportional hazards regression analysis. II. Accuracy and precision of regression estimates. J Clin Epidemiol 48:1503-1510

28. Miravitlles M, Espinosa C, FernandezLaso E, Martos JA, Maldonado JA, Gallego M (1999) Relationship between bacterial flora in sputum and functional impairment in patients with acute exacerbations of COPD. Study Group of Bacterial Infection in COPD. Chest 116:40-46

29. Tseng YC, Wang JT, Wu FL, Chen YC, Chie WC, Chang SC (2007) Prognosis of adult patients with bacteremia caused by extensively resistant Acinetobacter baumannii. Diagn Microbiol Infect Dis 59:181-190

30. Griffith DE, Brown-Elliott BA, Langsjoen B, Zhang Y, Pan X, Girard W, Nelson K, Caccitolo J, Alvarez J, Shepherd S, Wilson R, Graviss EA, Wallace RJ Jr (2006) Clinical and molecular analysis of macrolide resistance in Mycobacterium avium complex lung disease. Am J Respir Crit Care Med 174:928-934
31. Hsu DI, Okamoto MP, Murthy R, Wong-Beringer A (2005)

Fluoroquinolone-resistant Pseudomonas aeruginosa: risk factors for acquisition and impact on outcomes. J Antimicrob Chemother 55:535-541

32. Freeman J, Morris A, Blackmore T, Hammer D, Munroe S, McKnight L (2007) Incidence of nontuberculous mycobacterial disease in New Zealand, 2004. N Z Med J 120:U2580

33. O’Brien RJ, Geiter LJ, Snider DE Jr (1987) The epidemiology of nontuberculous mycobacterial diseases in the United States. Results from a national survey. Am Rev Respir Dis 135:1007-1014

34. Subcommittee of the Joint Tuberculosis Committee of the British Thoracic Society (2000) Management of opportunist mycobacterial infections: Joint Tuberculosis Committee Guidelines 1999. Subcommittee of the Joint Tuberculosis Committee of the British Thoracic Society. Thorax 55:210-218

35. Tsukamura M, Kita N, Shimoide H, Arakawa H, Kuze A (1988) Studies on the epidemiology of nontuberculous mycobacteriosis in Japan. Am Rev Respir Dis 137:1280-1284

36. Griffith DE, Girard WM, Wallace RJ Jr (1993) Clinical features of pulmonary disease caused by rapidly growing mycobacteria. An analysis of 154 patients. Am Rev Respir Dis 147:12711278
37. Han D, Lee KS, Koh WJ, Yi CA, Kim TS, Kwon OJ (2003) Radiographic and CT findings of nontuberculous mycobacterial pulmonary infection caused by Mycobacterium abscessus. AJR Am J Roentgenol 181:513-517

38. El-Solh AA, Nopper J, AbdulKhoudoud MR, Sherif SM, Aquilina AT, Grant BJ (1998) Clinical and radiographic manifestations of uncommon pulmonary nontuberculous mycobacterial disease in AIDS patients. Chest 114:138-145

39. Kobashi Y, Yoshida K, Miyashita N, Niki Y, Oka M (2006) Relationship between clinical efficacy of treatment of pulmonary Mycobacterium avium complex disease and drug-sensitivity testing of Mycobacterium avium complex isolates. J Infect Chemother 12:195-202

40. Marras TK, Morris A, Gonzalez LC, Daley CL (2004) Mortality prediction in pulmonary Mycobacterium kansasii infection and human immunodeficiency virus. Am J Respir Crit Care Med 170:793-798 